\title{
Profile of evolocumab and its potential in the treatment of hyperlipidemia
}

\author{
This article was published in the following Dove Press journal: \\ Drug Design, Development and Therapy \\ 15 June 2015 \\ Number of times this article has been viewed
}

\section{Arrigo FG Cicero \\ Alessandro Colletti \\ Claudio Borghi}

Medical and Surgical Sciences Department, University of Bologna, Bologna, Italy
Correspondence: Arrigo FG Cicero Atherosclerosis Research Center, Poliambulatorio Pad 2, Via Albertoni I5, 40138 Bologna, Italy

Tel +3905I 6362224

Fax +39 05I 6826125

Email arrigo.cicero@unibo.it
Abstract: Despite the proven efficacy of statins, they often fail to achieve low-density lipoprotein (LDL) cholesterol goals, especially in high-risk patients. Moreover, a large number of subjects cannot tolerate statins or full doses of these drugs, in particular patients with familial hypercholesterolemia. Thus, there is a need for additional effective LDL cholesterol-reducing agents. Evolocumab (AMG145) is a monoclonal antibody inhibiting proprotein convertase subtilisin/kexin type 9 that binds to the liver LDL receptor and prevents it from normal recycling by targeting it for degradation. Phase I, II, and III trials revealed that, on subcutaneous injection, either alone or in combination with statins, evolocumab is able to reduce high LDL cholesterol levels from $54 \%$ to $80 \%$, apolipoprotein B100 from $31 \%$ to $61 \%$, and lipoprotein(a) from $12 \%$ to $36 \%$, in a dose-dependent manner. The incidence of side effects seems to be low and mainly limited to nasopharyngitis, injection site pain, arthralgia, and back pain. Evolocumab is an innovative powerful lipid-lowering drug, additive to statins and/or ezetimibe, with a large therapeutic range associated with a low rate of mild adverse events. If the available data are confirmed in long-term trials with strong outcome measures, evolocumab will become an essential tool in the treatment of a large number of high-risk patients, such as those affected by familial hypercholesterolemia, those who are unable to tolerate an efficacious statin dosage, and those at very high cardiovascular risk and unable to achieve their target LDL cholesterol levels with currently available lipid-lowering therapies.

Keywords: hypercholesterolemia, lipid-lowering drugs, proprotein convertase subtilisin/kexin type 9 , AMG145

\section{Introduction}

Serum low-density lipoprotein (LDL) cholesterol levels seem to be the only reversible risk factor for cardiovascular disease, pharmacological reduction of which is not associated with a limit beyond which increased risk to health is found. ${ }^{1}$ The paradigm of "the lower, the better" acting simultaneously on different pharmacological targets, has recently been confirmed by the results of the IMPROVE-IT trial, where simultaneous use of statins (inhibitors of liver cholesterol synthesis) and ezetimibe (inhibitor of cholesterol absorption from the bowel) led to a dramatic decrease in LDL cholesterol and a parallel decrease in the risk of cardiovascular disease. ${ }^{2}$ However, despite this evidence, a large number of patients are not able to reach the desired LDL cholesterol target, mainly because of intolerance to standard treatment or very high baseline values related to genetic diseases of lipid metabolism. ${ }^{3}$ In this context, the search for new drugs with different mechanisms of action could improve control of LDL cholesterol in patients at increased risk of developing cardiovascular disease.

Proprotein convertase subtilisin/kexin type 9 (PCSK9) is a regulatory serine protease that binds to the epidermal growth factor-like repeat A domain of the LDL 
receptor, inducing its degradation (Figure 1). ${ }^{4}$ PCSK9 is synthesized primarily in the liver, then enters the circulation and binds to the hepatic LDL receptor, thus reducing the ability of the liver to remove LDL cholesterol. Therefore, increased activity of PCSK9 results in high LDL cholesterol levels, as shown in patients with gain-of-function mutations of this protease, ${ }^{5}$ whereas loss-of-function mutations are associated with low LDL cholesterol levels and a reduced risk of coronary heart disease. ${ }^{6}$ Low intracellular cholesterol levels (eg, in response to statin treatment) lead to activation of sterol regulatory element-binding protein-2, which causes coexpression of the LDL receptor and PCSK9; it is thought (albeit not clinically demonstrated as yet) that this coexpression could reduce the therapeutic effect of statins. ${ }^{7,8}$ On the basis of this evidence, specific human monoclonal antibodies directed against PCSK9 have now been developed, ie, 1D05-IgG2, evolocumab (AMG145), and alirocumab (REGN727/SAR236553). Phase II studies showed evolocumab and alirocumab to be effective in reducing LDL cholesterol from $40 \%$ to $80 \% .{ }^{9}$ Recent data from the first Phase III studies of PCSK9 inhibitors show LDL cholesterol reduction ranging from $54 \%$ to $80 \% .{ }^{10}$ This review summarizes the available clinical evidence on the efficacy and safety of evolocumab (AMG145), for which a relatively large amount of short-term and middle-term clinical data are currently available.

\section{Chemistry and pharmacodynamics}

Evolocumab $\left(\mathrm{C}_{6242} \mathrm{H}_{9648} \mathrm{~N}_{1668} \mathrm{O}_{1996} \mathrm{~S}_{56}\right)$ is a $141.8 \mathrm{kDa}$, fully human monoclonal immunoglobulin G2-lambda, with a gamma 2 heavy chain (1-441) [human VH (Homo sapiens IGHV1-18*01 (93.9\%)-(IGHD)-IGHJ6*01) [8.8.8] (1-115)Homo sapiens IGHG2*01 (116-441)] (129-214')-disulfide and lambda light chain $\left(1^{\prime}-215^{\prime}\right)$ [human V-LAMBDA (Homo sapiens IGLV2-14*01 (95.9\%)-IGLJ2*01 [9.3.9] (1'-109')IGLC2*01 $\left.\left(110^{\prime}-215^{\prime}\right)\right]$. It binds to the LDL receptor binding domain of PCSK9 with high affinity ( $\mathrm{kDa}<100 \mathrm{pM})$, strongly inhibiting its activity. ${ }^{11}$ As a consequence, administration of evolocumab is associated with a rapid (within 14 days) and impressive dose-dependent reduction of LDL cholesterol, with a parallel reduction in apolipoprotein B100 (ApoB) levels. ${ }^{12,13}$

\section{Pharmacokinetics}

Evolocumab is administered subcutaneously. The drug exhibits nonlinear pharmacokinetics at a dose of 21-420 $\mathrm{mg}$, meaning that the drug concentration in plasma does not increase strictly proportionally to the administered dose. Clearance can be described by parallel linear and nonlinear clearance pathways, ie, the $\mathrm{pK}$ for evolocumab reaches linearity at single doses $>210 \mathrm{mg}$ injected subcutaneously, with peak concentrations reached at 72 hours. Repeated doses above $140 \mathrm{mg}$ exhibited linear kinetics. Again, in Phase II and Phase III studies, the kinetics of evolocumab follow an approximately linear profile at doses $>140 \mathrm{mg}$ every 2 weeks, when administered alone or with statins. ${ }^{14}$

\section{Clinical efficacy}

In a Phase Ia study, 56 healthy subjects were randomized to receive evolocumab $(n=42)$ or placebo $(n=14),{ }^{15}$ and a mean LDL cholesterol decrease of up to $64 \%$ was found when compared with placebo $(P<0.0001)$. In a Phase Ib study, 42 hypercholesterolemic patients receiving statin treatment and 14 patients on placebo were enrolled, with the finding of a mean LDL cholesterol decrease of up to $81 \%$ versus placebo. Both studies showed that doses $>20 \mathrm{mg}$ start to have an effect on LDL cholesterol and that doses $>420 \mathrm{mg} /$ every 4 weeks lead to an LDL cholesterol reduction of over $60 \%$. Lipoprotein(a) [Lp(a)] decreased by up to $38 \%$ $(P<0.001)$ and ApoB decreased by up to $59 \%(P<0.001)$ in the Phase Ib trial. ${ }^{13}$ The mechanism by which evolocumab

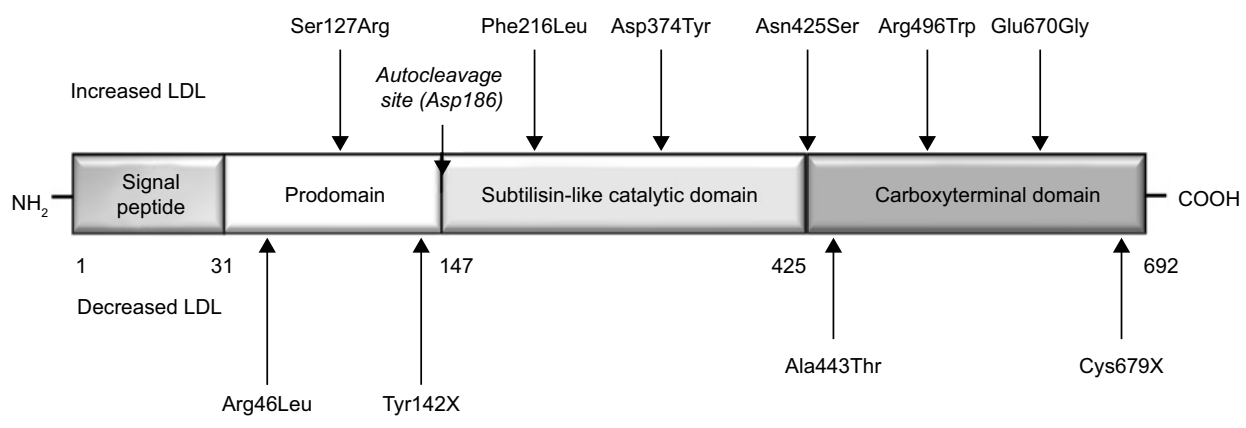

Figure I PCSK9 protein and its variants.

Abbreviations: PCSK9, proprotein convertase subtilisin/kexin type 9; LDL, low-density lipoprotein. 
reduces Lp(a) is not known; however, evolocumab probably could induce $\mathrm{Lp}$ (a) clearing via the LDL receptor or very low-density lipoprotein receptors.

In the Phase II MENDEL trial, 406 patients not treated with statins who had LDL cholesterol levels of 100-190 mg/dL, triglycerides $<400 \mathrm{mg} / \mathrm{dL}$, and Framingham risk scores $\leq 10 \%$ were randomized to: evolocumab $70 \mathrm{mg}, 105 \mathrm{mg}$, or $140 \mathrm{mg}$, or placebo every 2 weeks; evolocumab $280 \mathrm{mg}, 350 \mathrm{mg}$, or $420 \mathrm{mg}$, or placebo every 4 weeks; or oral ezetimibe $10 \mathrm{mg}$ / day. ${ }^{16}$ Evolocumab significantly reduced LDL cholesterol in all groups. Changes from baseline LDL cholesterol level were dose-dependent, ranging from $-41 \%(95 \% \mathrm{CI}-46,-36)$ to $-51 \%(\mathrm{CI}-56,-46)$ with administration of evolocumab every 2 weeks, and from $-39 \%(\mathrm{CI}-44,-34)$ to $-48 \%(-53,-43)$ with evolocumab every 4 weeks $(P<0.0001$ for all doses versus placebo or ezetimibe).

The Phase III MENDEL-2 trial compared the effect of AMG145 (140 mg every 2 weeks or $420 \mathrm{mg}$ monthly) with placebo and ezetimibe in hypercholesterolemic patients. ${ }^{17}$ A total of 614 patients with fasting LDL cholesterol $\geq 100$ $\mathrm{mg} / \mathrm{dL}$ and $<190 \mathrm{mg} / \mathrm{dL}$ and Framingham risk scores $\leq 10 \%$ were randomized. Evolocumab reduced LDL cholesterol from baseline by $55 \%-57 \%$ more than placebo and $38 \%-40 \%$ more than ezetimibe $(P<0.001)$. Approximately $70 \%$ of the patients on evolocumab achieved an LDL cholesterol level $<70 \mathrm{mg} / \mathrm{dL}$ compared with $\sim 1.5 \%$ of those on ezetimibe and $\sim 0.5 \%$ on placebo. Evolocumab also significantly decreased ApoB, triglycerides, Lp(a), and non-high-density lipoprotein (HDL) cholesterol levels (all $P<0.05$ ). HDL cholesterol concentrations were also significantly $(P<0.05)$ increased.

In the Phase II LAPLACE-TIMI 57 study, ${ }^{18} 631$ hypercholesterolemic patients were randomly assigned to evolocumab $70 \mathrm{mg}, 105 \mathrm{mg}$, or $140 \mathrm{mg}$, or matching placebo every 2 weeks; or to evolocumab $280 \mathrm{mg}, 350 \mathrm{mg}$, and $420 \mathrm{mg}$, and matching placebo every 4 weeks. At week 12 , the mean LDL cholesterol concentrations were dose-dependently reduced by evolocumab every 2 weeks (ranging from $41.8 \%$ to $66.1 \% ; P<0.0001$ for each dose versus placebo) and evolocumab every 4 weeks (ranging from $41.8 \%$ to $50.3 \%$; $P<0.0001) .{ }^{19}$ This study also confirmed a dose-dependent effect of evolocumab on $\mathrm{Lp}$ (a) level, proportional to the baseline level of $\mathrm{Lp}(\mathrm{a}),{ }^{20}$ ranging from $-18 \%$ to $-32.3 \%$. This effect was also observed with other PCSK9 antibodies, and is likely to be a class effect. ${ }^{21}$

The Phase III LAPLACE-2 study evaluated the efficacy and tolerability of evolocumab when used in combination with a moderate-intensity versus high-intensity statin in patients with primary hypercholesterolemia and mixed dyslipidemia. ${ }^{22}$ This 12-week, randomized, double-blind, placebo-controlled and ezetimibe-controlled trial enrolled 1,899 patients, who were randomized to compare evolocumab (140 mg subcutaneous every 2 weeks or $420 \mathrm{mg}$ subcutaneously monthly) with placebo subcutaneously (every 3 weeks or monthly) or ezetimibe (10 mg or placebo daily only for patients receiving atorvastatin) when added to statin therapies. Evolocumab reduced LDL cholesterol levels by $63 \%-75 \%$ versus placebo at a mean of weeks 10 and 12 in the moderate-intensity and high-intensity statin-treated groups. Evolocumab also reduced non-HDL cholesterol concentrations at a mean of weeks 10 and 12 by $58 \%-65 \%$, ApoB by $51 \%-59 \%$, and $\mathrm{Lp}$ (a) by $21 \%-36 \%$ (all versus placebo). Triglyceride concentrations were also decreased by $12 \%-30 \%$ in patients receiving evolocumab versus placebo, while HDL cholesterol levels were increased by $4 \%-10 \%$ versus placebo.

In the Phase II RUTHERFORD study, evolocumab was administered to statin-treated patients affected by heterozygous familial hypercholesterolemia, mainly because of loss-of-function mutations in the LDL receptor alleles (98\%) and less commonly in relation to a defect in ApoB or gain-offunction mutation in PCSK9. ${ }^{23,24}$ Evolocumab was tested at doses of $350 \mathrm{mg}$ and $420 \mathrm{mg}$ every 4 weeks in 168 patients, whereas other 56 patients were treated with placebo plus statin. ${ }^{25}$ Mean reduction in LD cholesterol was $43 \%$ in the $350 \mathrm{mg}$ group and 55\% in the $420 \mathrm{mg}$ group, compared with a $1 \%$ increase in the placebo group $(P<0.001)$. At week 12 , evolocumab $350 \mathrm{mg}$ every 4 weeks resulted in LDL cholesterol $<100 \mathrm{mg} / \mathrm{dL}$ in $70 \%$ of patients and evolocumab $420 \mathrm{mg}$ every 4 weeks reached this target value in $89 \%$ of patients. An LDL cholesterol target $<70 \mathrm{mg} / \mathrm{dL}$ was obtained in $44 \%$ and $65 \%$ of patients, respectively. LDL cholesterol changes were higher with evolocumab $420 \mathrm{mg}$ than with alirocumab monthly, with similar statin doses. ${ }^{26}$ A statistically significant dose-dependent reduction in triglycerides was also observed (15\% and 20\%, respectively), associated with an HDL cholesterol increase of approximately $7 \%$ and a dosedependent $\mathrm{Lp}$ (a) reduction ranging from $23 \%$ to $32 \%$.

The Phase III RUTHERFORD-2 trial investigated the relationship between the response of LDL cholesterol to evolocumab and genotype in subjects with heterozygous familial hypercholesterolemia identified on the basis of clinical criteria, on stable lipid-lowering therapy for $\geq 4$ weeks, and with fasting LDL cholesterol $\geq 100 \mathrm{mg} / \mathrm{dL} .{ }^{27}$ The patients were randomized 2:2:1:1 to receive evolocumab $140 \mathrm{mg}$ every 2 weeks, evolocumab $420 \mathrm{mg}$ monthly, placebo every 2 weeks, or placebo monthly for 12 weeks. 
Of the 331 randomized patients, 264 agreed to the genetic analysis, and of those, $80 \%$ had mutations known to cause familial hypercholesterolemia. Patients found to have an LDL receptor mutation were grouped by LDL receptor functional class as defective or negative. ${ }^{28,29}$ Evolocumab administered every 2 weeks, or monthly resulted in mean LDL cholesterol reductions at week 12 of $59 \%$ and $61 \%$, respectively, versus placebo $(P<0.001)$ : LDL cholesterol reductions with every 2 weeks or monthly dosing were $61 \%$ and $55 \%$ in those with an LDL receptor mutation associated with no function, $49 \%$ and $66 \%$ in those with defective function, and $62 \%$ and $63 \%$ where the LDL receptor status was unclassified. At week 12 , LDL cholesterol $<70 \mathrm{mg} / \mathrm{dL}$ was achieved by $68 \%$ and $63 \%$ of patients in the evolocumab $140 \mathrm{mg}$ every 2 weeks and 420 monthly groups, respectively, compared with $2 \%$ of patients in the placebo groups. Compared with placebo, mean reductions in Lp(a) were $32 \%$ and $28 \%$ at week 12 (both doses $P<0.001)$. Treatment with evolocumab $140 \mathrm{mg}$ every 2 weeks and $420 \mathrm{mg}$ monthly compared with placebo resulted in mean triglycerides decreases of $20 \%(P<0.001)$ and $12 \%$ $(P<0.05)$, respectively, at week 12 . HDL cholesterol was increased by $9 \%$ in both evolocumab groups.

In the Phase II GAUSS study, evolocumab was tested in statin-intolerant patients, comparing the effect of evolocumab at doses of $280 \mathrm{mg}, 350 \mathrm{mg}$, and $420 \mathrm{mg}$ with that of placebo/ ezetimibe. ${ }^{30}$ Statin intolerance was defined as inability to tolerate effective doses of at least two different statins because of myalgia, myopathy, myositis, or rhabdomyolysis that resolved after statin discontinuation. Maximal reduction in LDL cholesterol was observed within 2 weeks of treatment, with or without ezetimibe. At week 12, LDL cholesterol changes were dose-related, ranging from -67 to $-91 \mathrm{mg} / \mathrm{dL}$, increasing to $-110 \mathrm{mg} / \mathrm{dL}$ in the $420 \mathrm{mg}+$ ezetimibe group. Lp(a) levels were reduced by $20 \%-29 \%$ in the evolocumab-treated groups (all $P<0.001$ ). The increase in HDL cholesterol ranged from $6 \%$ to $12 \%$, compared with the placebo/ezetimibe group, with similar increases seen in ApoA1.

In the Phase III GAUSS-2 trial, researchers evaluated the efficacy and safety of evolocumab compared with oral ezetimibe in patients with hypercholesterolemia unable to tolerate effective statin doses $(-10 \%-20 \%$ of patients were receiving statins). ${ }^{31,32}$ The GAUSS-2 trial was a 12 -week, double-blind study, which enrolled 307 patients (mean age: $62 \pm 10$ years, mean baseline LDL cholesterol: $193 \pm 59 \mathrm{mg} / \mathrm{dL}$ ) on no statin or on a low-dose statin (weekly dose seven times the smallest available tablet strength). Mean percent reductions from baseline at a mean of weeks 10 and 12 were $56.1 \%$ with $140 \mathrm{mg}$ every 2 weeks and $55.3 \%$ with $420 \mathrm{mg}$ once monthly, corresponding to treatment differences versus ezetimibe of $36.9 \%$ and $38.7 \%$, respectively $(P<0.001)$, with similar mean percent reductions at week 12 $(P<0.001)$. Evolocumab-treated patients were more likely to achieve LDL cholesterol target levels than ezetimibe-treated patients $(\sim 76.5 \%$ versus $\sim 5.5 \%$, respectively). Compared with ezetimibe, evolocumab resulted in significant reductions in ApoB, Lp(a), non-HDL cholesterol concentrations, and ApoB/ApoA-I and total cholesterol/HDL cholesterol ratios $(P<0.001)$.

The Phase III DESCARTES trial evaluated the safety and efficacy of 52 weeks of treatment with evolocumab. ${ }^{33}$ Patients with an LDL cholesterol level $\geq 75 \mathrm{mg} / \mathrm{dL}$ were randomly assigned in a 2:1 ratio to receive either evolocumab (420 mg) or placebo every 4 weeks for 52 weeks. Nine hundred and one patients were included in the primary analysis. The reduction in LDL cholesterol from baseline in the evolocumab group, taking into account the change in the placebo group, was $57.0 \% \pm 2.1 \%(P<0.001)$. LDL cholesterol levels were reduced to below $70 \mathrm{mg} / \mathrm{dL}$ in $82.3 \%$ of patients in the evolocumab group, compared with $6.4 \%$ in the placebo group. Evolocumab also significantly decreased the concentrations of ApoB (44.2\%), non-HDL cholesterol (50.3\%), Lp(a) $(22.4 \%)$, and triglycerides $(11.5 \%)$. HDL cholesterol was significantly increased by $5.4 \%$.

In the Phase III TESLA Part B trial, 50 patients with homozygous familial hypercholesterolemia on stable lipidlowering therapy and not on lipoprotein apheresis received either subcutaneous evolocumab $420 \mathrm{mg}$ or placebo every 4 weeks for 12 weeks. ${ }^{34}$ Of the 50 patients randomized, 49 completed the study (16 in the placebo group and 33 in the evolocumab group). Evolocumab significantly reduced LDL cholesterol (as measured by ultracentrifugation) by $30.9 \%$ compared with placebo. These data confirm the results of a previous small trial carried out in patients with receptor-negative or receptor-defective homozygous familial hypercholesterolemia, in which eight patients treated with subcutaneous $420 \mathrm{mg}$ evolocumab every 4 weeks for $\geq 12$ weeks, followed by $420 \mathrm{mg}$ evolocumab every 2 weeks for 12 more weeks. At week 12, the mean change from baseline in LDL cholesterol was $-16.5 \%$ (range $5.2 \%$ to $-43.6 \% ; P<0.078$ ) and $-13.9 \%$ (range $39.9 \%$ to $-43.3 \% ; P<0.148$ ) with 4 -week and 2 -week dosing, respectively. While no LDL cholesterol reduction was seen in the two receptor-negative patients over the treatment period, the mean LDL cholesterol reduction in the six LDL receptor-defective patients was $19.3 \% \pm 16 \%$ and $26.3 \% \pm 20 \%$ with 4 -week and 2 -week dosing, respectively 
( $P=0.03$ for both values), ranging from $4 \%$ to $48 \%$ with the 2-week dosing regimen. ${ }^{35}$

The main characteristics of these studies are summarized in Tables 1-3. The OSLER trial was then started in October 2011 to evaluate whether long-term exposure to evolocumab is safe and well tolerated on a background of standard of care. More than 1,100 hypercholesterolemic participants were enrolled from the Phase II trials, and the study is planned to end in May 2016. ${ }^{36-38}$

Among the Phase III trials aiming to confirm the efficacy and safety of evolocumab in a specific subpopulation of hypercholesterolemic subjects, the main one is PROFICIO, while the FOURIER study, that started in January 2013 and will continue to 2018 , is evaluating whether evolocumab decreases cardiovascular events versus placebo in 22,500 patients with cardiovascular disease. ${ }^{39}$

\section{Safety and tolerability data}

Based on the available pharmacological data for evolocumab, we could foresee two main types of possible toxicity. Targetrelated toxicity includes the effects of antibodies in nonintended tissues. Modality-related toxicity includes acute hypersensitivity reactions. Another restricting point could be the formation of antibodies against murine and chimeric antibodies, ie, an antiantibody response, which is very low for fully humanized antibodies. The most safe/efficacious dosages of ascending doses of evolocumab have been tested in pharmacodynamic and pharmacokinetic studies performed in patients using stable doses of statins. ${ }^{40}$

No serious adverse events were observed in the Phase I studies of evolocumab, but myositis was reported in three patients, with creatine kinase elevations $>2.5-5$ times the upper limit of normal, which resolved in less than 4 weeks. $^{13}$

In the MENDEL study, nonserious adverse events occurred with a similar incidence in evolocumab-treated, placebo-treated, and ezetimibe-treated patients (46\%-58\%); no serious treatment-related adverse events were reported. ${ }^{16}$ Also, in the MENDEL-2 trial, ${ }^{17}$ nonserious adverse events occurred with a similar incidence in evolocumab-treated, placebo-treated, and ezetimibe-treated patients (44\%-46\%). Serious adverse events occurred in $1.3 \%$ of the patients receiving AMG145 versus $0.6 \%$ in both the placebo and ezetimibe groups.

The LAPLACE-TIMI 57 study evaluated different doses of evolocumab together with statin \pm ezetimibe, but no serious adverse events occurred. Treatment-related adverse events were similar in the evolocumab and placebo groups; none of these events was severe. ${ }^{18,19}$ In the LAPLACE II trial, nonserious adverse events occurred with a similar incidence in evolocumab-treated, placebo-treated, and ezetimibetreated patients $(36 \%-40 \%)$, with back pain, arthralgia, headache, muscle spasms, and pain in the extremities being the most common adverse events in the evolocumab-treated patients. ${ }^{41}$

In the RUTHERFORD study, ${ }^{23,24}$ the most common treatment-related adverse events were nasopharyngitis (12.5\% with evolocumab $350 \mathrm{mg}, 12.7 \%$ with evolocumab $420 \mathrm{mg}$, and $10.7 \%$ with placebo), injection site pain $(9.1 \%$ with $350 \mathrm{mg}, 3.6 \%$ with $420 \mathrm{mg}$, and $1.8 \%$ with placebo), and headache (5.5\% with $350 \mathrm{mg}, 5.4 \%$ with $420 \mathrm{mg}$, and $8.9 \%$ with placebo). Nasopharyngitis was thought to be caused by an immune-mediated process, but the exact underlying pathogenetic mechanism has to be fully clarified. Only one patient treated with evolocumab $420 \mathrm{mg}$ experienced creatine kinase increase more than ten times the upper limit of normal without symptoms. An increase in transaminases more than three times (but less than five times) the upper limit of normal was observed in two patients treated with evolocumab. In the RUTHERFORD-2 trial, the incidence of nonserious and serious adverse events was comparable with rates reported in previous studies of evolocumab. ${ }^{42}$

In the GAUSS study, the most common treatment-related adverse effect was myalgia, which occurred at the highest incidence in 5\% of the $280 \mathrm{mg}$ group and $20 \%$ in the evolocumab/ ezetimibe group. ${ }^{40}$ Registered serious adverse events were coronary artery disease, acute pancreatitis, hip fracture, and syncope, but they were considered not related to the tested lipidlowering treatment. Two patients experienced creatine kinase levels higher than ten times the upper limit of normal during the study, but values normalized after treatment cessation.

In the GAUSS II trial, muscle-related adverse effects occurred in $12 \%$ of evolocumab-treated patients and $23 \%$ of ezetimibe-treated patients. Myalgia occurred in $8 \%$ of evolocumab-treated patients and $18 \%$ of ezetimibe-treated patients. Patients using low-dose statin therapy were more likely to develop myalgia, both in the ezetimibe group (statin versus no statin, $21 \%$ versus $17 \%$ ) and the evolocumab group (statin versus no statin, $17 \%$ versus $6 \%$ ). ${ }^{43}$

In the OSLER trial, adverse events occurred in $81.4 \%$ of patients treated with evolocumab and in $73.1 \%$ of those in the standard treatment group. The five most common adverse events in the evolocumab group compared to the standard group were nasopharyngitis (12.2\% versus $9.8 \%)$, upper respiratory tract infections $(7.7 \%$ versus $7.6 \%)$, influenza ( $7.1 \%$ versus $5.2 \%)$, arthralgia (6.9\% versus $4.3 \%)$, and 


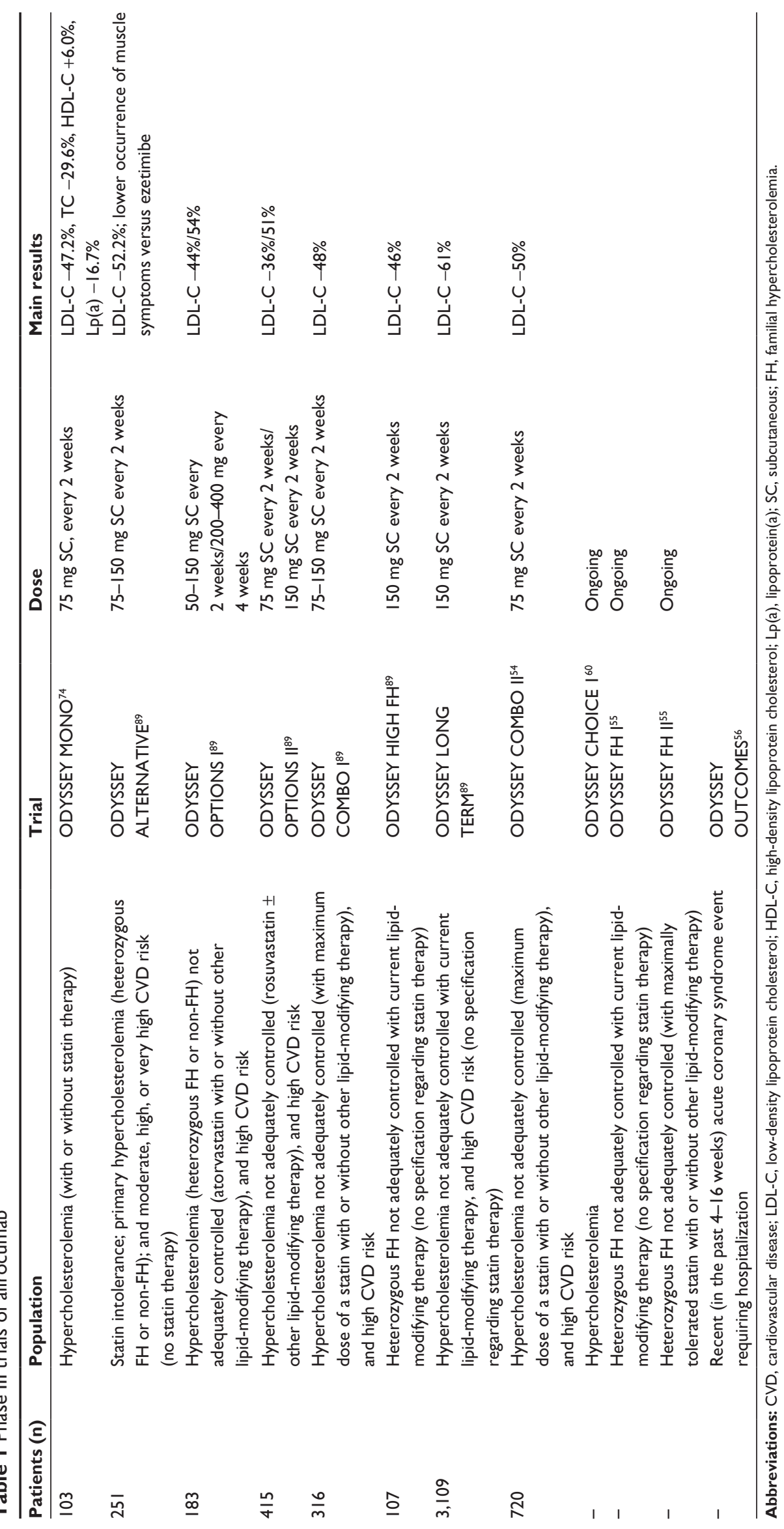




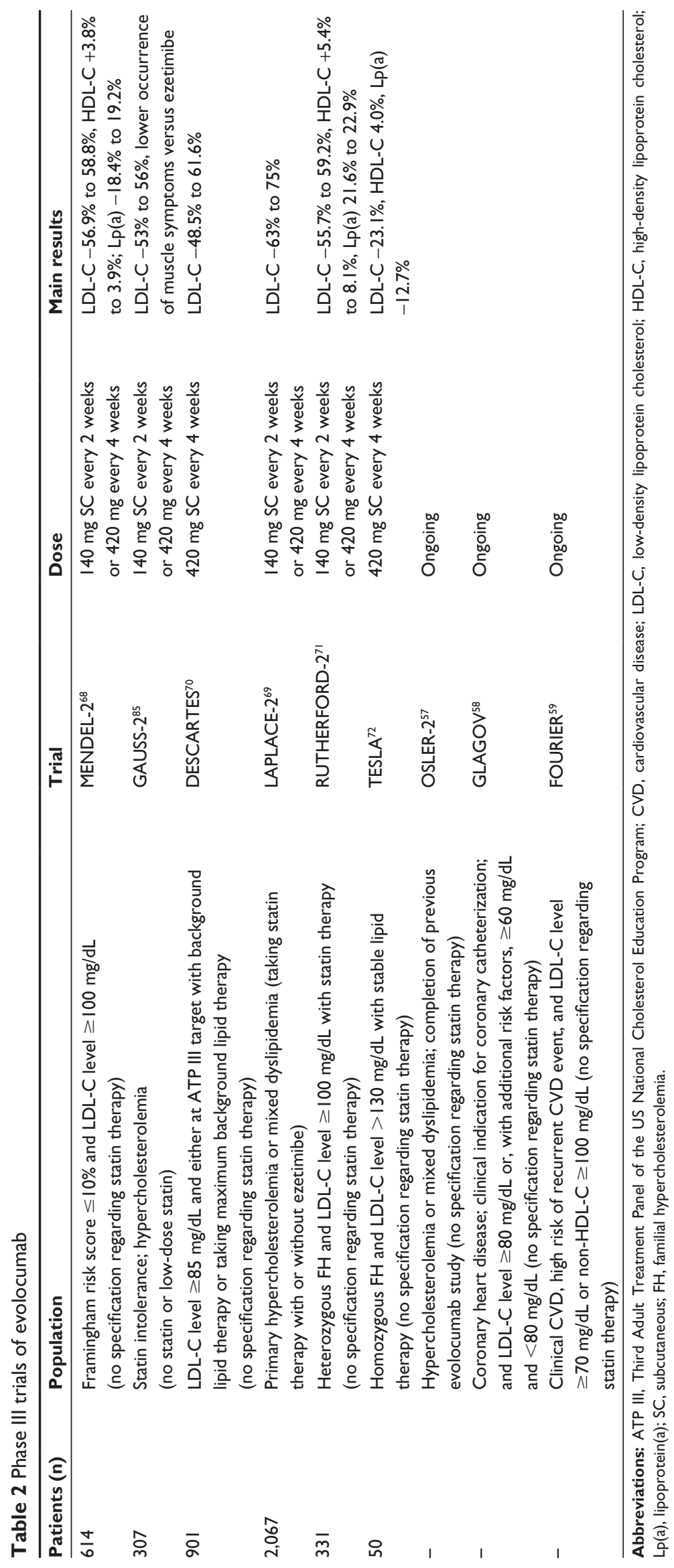


Table 3 Phase III trials of bococizumab

\begin{tabular}{|c|c|c|c|}
\hline Patients (n) & Population & Trial & Main results \\
\hline- & $\begin{array}{l}\text { Heterozygous FH; high or very high CVD risk; LDL-C level }>70 \mathrm{mg} / \mathrm{dL} \text { and TG level } \leq 400 \mathrm{mg} / \mathrm{dL} \\
\text { (with statin therapy) }\end{array}$ & SPIRE-HF & Ongoing \\
\hline- & High or very high CVD risk; LDL-C level $>70 \mathrm{mg} / \mathrm{dL}$ and TG level $\leq 400 \mathrm{mg} / \mathrm{dL}$ (with statin therapy) & SPIRE-HR & Ongoing \\
\hline- & High or very high CVD risk; LDL-C level $>70 \mathrm{mg} / \mathrm{dL}$ and TG level $\leq 400 \mathrm{mg} / \mathrm{dL}$ (with statin therapy) & SPIRE-LDL & Ongoing \\
\hline- & $\begin{array}{l}\text { High CVD risk; LDL-C level } \geq 70 \mathrm{mg} / \mathrm{dL} \text { and }<100 \mathrm{mg} / \mathrm{dL} \text {, or non-HDL-C level } \geq 100 \mathrm{mg} / \mathrm{dL} \\
\text { and }<130 \mathrm{mg} / \mathrm{dL} \text {, with lipid-lowering therapy (no specification regarding statin therapy) }\end{array}$ & SPIRE-I & Ongoing \\
\hline- & $\begin{array}{l}\text { High CVD risk; LDL-C level } \geq 100 \mathrm{mg} / \mathrm{dL} \text { or non-HDL-C level } \geq 130 \mathrm{mg} / \mathrm{dL} \text {, with lipid-lowering } \\
\text { therapy (no specification regarding statin therapy) }\end{array}$ & SPIRE-2 & Ongoing \\
\hline
\end{tabular}

Abbreviations: FH, familial hypercholesterolemia; CVD, cardiovascular disease; LDL-C, low-density lipoprotein cholesterol; HDL-C, high-density lipoprotein cholesterol; Lp(a), lipoprotein(a); SC, subcutaneous; TG, triglycerides.

back pain (6.5\% versus 5.4\%). Other reported adverse events included muscle-related events ( $9.2 \%$ versus $9.8 \%)$, elevated liver function tests $(1.8 \%$ versus $1.6 \%)$, and elevated creatine kinase (1.0\% versus $1.9 \%)$. Serious adverse events occurred in a similar percentage in patients treated with evolocumab $(7.1 \%)$ and in those in the standard care group $(6.3 \%))^{37,38}$

In the DESCARTES trial, the overall incidence of adverse events occurring during treatment was similar in the evolocumab group (74.8\%) and the placebo group (74.2\%). The most common adverse events in the evolocumab group were nasopharyngitis, upper respiratory tract infection, influenza, and back pain. ${ }^{33}$ In the TESLA Part B trial, adverse events occurred in $63 \%$ of the patients in the placebo group and in $36 \%$ of the patients in the evolocumab group. No serious adverse events occurred, and no anti-evolocumab antibody development was detected during the study. ${ }^{34}$

\section{Discussion}

In clinical practice, reduction of LDL cholesterol levels is usually achieved by use of reversible inhibitors of 3-hydroxy3-methylglutaryl-coenzyme A reductase (statins), also associated with inhibitors of the intestinal absorption of cholesterol (ionic exchange resins, such as cholestyramine or colestimide, colestipol, or ezetimibe). ${ }^{44}$ This strategy is usually effective and relatively well tolerated; however, subjects at high risk for cardiovascular disease, ie, those requiring more intensive treatment for reduction of hypercholesterolemia, often do not reach the desired LDL cholesterol target, either because of lack of efficacy of the available therapies or because of side effects of the high drug dosages required. ${ }^{45,46}$

In this context, an overall well-tolerated new treatment option, enlarging and empowering our ability to manage high-risk patients, especially when statin-intolerant (at least according to the definition used in the above cited trials), could be a great innovation in therapy. ${ }^{47}$ This is clearly useful when following the indications given by European Society of Cardiology/European Atherosclerosis Society guidelines, ${ }^{48}$ which focus their attention on baseline cholesterol levels and use a targeted approach, the LDL-C target seems to be less relevant when considering American College of Cardiology/American Heart Association guidelines, which have a statin-centered approach independent of baseline cholesterol levels and a specific LDL cholesterol target to be reached. ${ }^{49}$ Moreover, evolocumab positively modulates the whole lipid pattern, significantly decreases the Lp(a) level, ${ }^{50}$ a genetically determined lipid risk factor, and other lipid and lipoprotein fractions such as $\mathrm{ApoB}$, triglycerides, and non-HDL cholesterol, while increasing the HDL cholesterol level. Due to the strong LDL cholesterol-lowering achieved with evolocumab, levels of LDL cholesterol well below 50 $\mathrm{mg} / \mathrm{dL}$ will be observed in a large number of patients treated with these agents. ${ }^{10}$

Even if the clinical relevance of the $L p(a)$-reducing effect has to be tested in further trials, it could be really useful, especially considering the scarcity of drugs acting on $\mathrm{Lp}(\mathrm{a}) .{ }^{51}$ What is even more encouraging is that similar efficacy and safety data have been confirmed also with alirocumab, another independently developed human antiPCKS9 antibody..$^{52,53}$

In summary, evolocumab is a powerful lipid-lowering drug, with an apparently large dose-dependent therapeutic range associated with a low rate of usually mild adverse events. Its efficacy seems to be evident both when administered alone and when given in association with statins and/ or ezetimibe. If the available data are confirmed in long-term trials with strong outcomes, evolocumab will provide an essential tool to efficiently treat high risk patients who need to reach ambitious LDL cholesterol targets.

\section{Disclosure}

The authors report no conflicts of interest in this work. 


\section{References}

1. Cholesterol Treatment Trialists' (CTT) Collaboration. Efficacy and safety of LDL-lowering therapy among men and women: analysis of individual data from 174000 participants in 27 randomized trials. Lancet. 2015;385:1397-1405.

2. Kohno T. Report of the American Heart Association (AHA) Scientific Session 2014, Chicago. Circ J. 2014;79:34-40.

3. Reiner Z, De Bacquer D, Kotseva K, et al. Treatment potential for dyslipidaemia management in patients with coronary heart disease across Europe: findings from the EUROASPIRE III survey. Atherosclerosis. 2013;231:300-307.

4. Maxwell KN, Breslow JL. Adenoviral mediated expression of PCSK9 in mice results in a low-density lipoprotein receptor knockout phenotype. Proc Natl Acad Sci U S A. 2004;101:7100-7105.

5. Cohen JC, Boerwinkle E, Mosley TH Jr, Hobbs HH. Sequence variations in dominant hypercholesterolemia. Nat Genet. 2003;34 154-156.

6. Abifadel M, Varret M, Rabee JD, et al. Mutations in PCSK9 cause autosomal PCSK9, low LDL, and protection against coronary heart disease. N Engl J Med. 2006;354:1264-1267.

7. Urban D, Pöss J, Böhm M, Laufs U. Targetting the proprotein convertase subtisilin/kexin type 9 for the treatment of dyslipidemia and atherosclerosis. J Am Coll Cardiol. 2013;62:1401-1408.

8. Rashid S, Curtis DE, Garuti R, et al. Decreased plasma cholesterol and hypersensitivity to statins in mice lacking PCSK9. Proc Natl Acad Sci US A. 2005;102:5374-5379.

9. Farnier M. PCSK9 inhibitors. Curr Opin Lipidol. 2013;24:251-258.

10. Gouni-Berthold J, Berthold HK. PCSK9 antibodies for the treatment of hypercholesterolemia. Nutrients. 2014;6:5517-5533.

11. Stein EA, Wasserman SM, Dias C, Scott R, Raal F. AMG145. Drugs Fut. 2013;38:451-459.

12. Chan CY, Piper DE, Cao Q, et al. A proprotein convertase subtilisin/ kexin type neutralizing antibody reduces serum cholesterol in mice and non human primates. Proc Natl Acad Sci U S A. 2009;106: 9820-9825.

13. Cicero AF, Tartagni E, Ertek S. Efficacy and safety profile of evolocumab (AMG145), an injectable inhibitor of the proprotein convertase subtilisin/kexin type 9: the available clinical evidence. Exp Opin Biol Ther. 2014;14:863-868.

14. Foltz IN, Karow M, Wasserman SM. Evolution and emergence of therapeutic monoclonal antibodies. What cardologists need to know. Circulation. 2013;127:2222-2230.

15. Koren MJ, Scott R, Kim JB, et al. Efficacy, safety, and tolerability of a density lipoprotein cholesterol levels. Results from 2 randomized, double-blind, placebo-controlled, ascending dose phase 1 studies in healthy volunteers and hypercholesterolemic subjects on statins. $J \mathrm{Am}$ Coll Cardiol. 2012;60:1888-1898.

16. Dias CS, Shaywitz AJ, Wasserman SM, et al. Effects of AMG145 on lowmonoclonal antibody to proprotein convertase subtilisin/kexin type 9 as monotherapy in patients with hypercholesterolaemia (MENDEL): a randomised, double-blind, placebo-controlled, phase 2 study. Lancet. 2012;380:1995-2006.

17. Koren MJ, Lundqvist $P$, Bolognese $M$, et al. Anti-PCSK9 monotherapy for hypercholesterolemia - the MENDEL-2 randomized, controlled phase 3 clinical trial of evolocumab. J Am Coll Cardiol. 2014;63: 2531-2540.

18. Kohli P, Desai NR, Giugliano RP, et al. Design and rationale of the LAPLACE-TIMI 57 trial: a phase II, double-blind, placebo-controlled study of the efficacy and tolerability of a monoclonal antibody inhibitor of PCSK9 in subjects with hypercholesterolemia on background statin therapy. Clin Cardiol. 2012;35:385-391.

19. Giugliano RP, Desai NR, Kohli P, et al. Efficacy, safety, and tolerability of a monoclonal antibody to proprotein convertase subtilisin/kexin type 9 in combination with a statin in patients with hypercholesterolaemia (LAPLACE-TIMI 57): a randomised, placebo-controlled, dose-ranging, phase 2 study. Lancet. 2012;380:2007-2017.
20. Desai NR, Kohli P, Giugliano RP, et al. AMG145, a monoclonal antibody against proprotein convertase subtilisin kexin type 9 , significantly reduces lipoprotein(a) in hypercholesterolemic patients receiving statin therapy: an analysis from the LDL-C Assessment with Proprotein Convertase Subtilisin Kexin Type 9 Monoclonal Antibody Inhibition Combined with Statin Therapy (LAPLACE)-Thrombolysis in Myocardial Infarction (TIMI) 57 trial. Circulation. 2013;128: 962-969.

21. McKenney JM, Koren MJ, Kereiakes DJ, et al. Safety and efficacy of a monoclonal antibody to proprotein convertase subtilisin/kexin type 9 serine protease, SAR236553/REGN727, in patients with primary hypercholesterolemia receiving ongoing stable atorvastatin therapy. $J$ Am Coll Cardiol. 2012;59:2344-2353.

22. Robinson JG, Nedergaard BS, Rogers WJ, et al. Effect of evolocumab or ezetimibe added to moderate- or high-intensity statin therapy on LDL-C lowering in patients with hypercholesterolemia: the LAPLACE-2 randomized clinical trial. JAMA. 2014;311:1870-1882.

23. Leigh SE, Foster AH, Whittall RA, et al. Uptake and analysis of the University College London low density lipoprotein receptor familial hypercholesterolemia database. Ann Hum Genet. 2008;72:485-498.

24. Innerarity TL, Weisgraber KH, Arnold KS, et al. Familial defective apolipoprotein B-100: low density lipoproteins with abnormal receptor binding. Proc Natl Acad Sci U S A. 1987;84:6919-6923.

25. Raal F, Scott R, Somaratne R, et al. Low-density lipoprotein cholesterollowering effects of AMG 145, a monoclonal antibody to proprotein convertase subtilisin/kexin type 9 serine protease in patients with heterozygous familial hypercholesterolemia: the Reduction of LDL-C with PCSK9 Inhibition in Heterozygous Familial Hypercholesterolemia Disorder (RUTHERFORD) randomized trial. Circulation. 2012;126:2408-2417.

26. Stein EA, Gipe D, Bergeron J, et al. Effect of a monoclonal antibody to protein convertase subtilisin/kexin type 9 serine protease, SAR236553/ REGN727, in patients with primary hypercholesterolemia receiving ongoing stable atorvastatin therapy. J Am Coll Cardiol. 2012;59: 2344-2353.

27. Raal F, Stein E, Dufour, et al. PCSK9 inhibition with evolocumab (AMG 145) in heterozygous familial hypercholesterolemia (RUTHERFORD-2): a randomised, double-blind, placebo-controlled trial. Lancet. 2014;385:331-340.

28. Hobbs HH, Brown MS, Goldstein JL. Molecular genetics of the LDL receptor gene in familial hypercholesterolemia. Hum Mutat. 1992;1:445-466.

29. Usifo E, Leigh SE, Whittall RA, et al. Low-density lipoprotein receptor gene familial hypercholesterolemia variant database: update and pathological assessment. Ann Hum Genet. 2012;76:387-401.

30. Sullivan D, Olsson AG, Scott R, et al. Effect of a monoclonal antibody to PCSK9 on low-density lipoprotein cholesterol levels in statin-intolerant patients. The GAUSS Randomized Trial. JAMA. 2012;308:2497-2506.

31. Stroes E, Colquhoun D, Sullivan D, et al. Anti-PCSK9 antibody effectively lowers cholesterol in patients with statin intolerance: the GAUSS-2 randomized, placebo-controlled phase 3 clinical trial of evolocumab. J Am Coll Cardiol. 2014;63:2541-2548.

32. Zhang H, Plutzky J, Skentzos S, et al. Discontinuation of statins in routine care settings: a cohort study. Ann Intern Med. 2013;158:526-534.

33. Blom DJ, Hala T, Bolognese M, et al. A 52-week placebo-controlled trial of evolocumab in hyperlipidemia. N Engl J Med. 2014;370: 1809-1819.

34. Raal FJ, Honarpour N, Blom DJ, et al; for the TESLA Investigators. Inhibition of PCSK9 with evolocumab in homozygous familial hypercholesterolemia (TESLA Part B): a randomised, double-blind, placebo-controlled trial. Lancet. 2014;385:341-350.

35. Stein EA, Honarpour N, Wasserman SM, Xu F, Scott R, Raal FJ. Effect of the proprotein convertase subtilisin/kexin 9 monoclonal antibody, AMG 145, in homozygous familial hypercholesterolemia. Circulation. 2013;128:2113-2120. 
36. Amgen. Open label study of long term evaluation against LDL-C trial (OSLER). Available from: http://clinicaltrials.gov/show/NCT01439880. Accessed February 1, 2014.

37. Koren MJ, Giugliano RP, Raal F, et al. Randomized comparison of the safety, tolerability and efficacy of long-term administration of AMG 145 versus standard of care in 1004 patients: 52 week results from the OSLER trial. Circulation. 2014;129:234-243.

38. Mearns BM. Dyslipidaemia: 1-year results from OSLER trial of antiPCSK9 monoclonal antibody evolocumab. Nat Rev Cardiol. 2014; $11: 63$.

39. Amgen. Further cardiovascular outcomes research with PCSK9 inhibition in subjects with elevated risk (FOURIER). Available from: http:// clinicaltrials.gov/show/NCT01764633. Accessed February 1, 2014.

40. Amgen. Multiple dose study to evaluate the safety, tolerability, pharmacokinetics and pharmacodynamics of AMG 145 in subjects with hyperlipidemia on stable doses of a statin. Available from: http:// clinicaltrials.gov/ct2/show/record/NCT01133522. Accessed February 1, 2014.

41. Robinson JG, Nedergaard BS, Rogers WJ, et al. Effect of evolocumab or ezetimibe added to moderate- or high-intensity statin therapy on LDL-C lowering in patients with hypercholesterolemia: The LAPLACE-2 randomized clinical trial. JAMA. 2014;311:1870-1882.

42. Raal F, Stein E, Dufour R, et al. PCSK9 inhibition with evolocumab (AMG 145) in heterozygous familial hypercholesterolemia (RUTHERFORD-2): a randomised, double-blind, placebo-controlled trial. Lancet. 2014;385:331-340.

43. Stroes E, Colquhoun D, Sullivan D, et al. Anti-PCSK 9 antibody effectively lowers cholesterol in patients with statin intolerance: the GAUSS-2 randomized, placebo-controlled phase 3 clinical trial of evolocumab. J Am Coll Cardiol. 2014;63:2541-2548.

44. Gotto AM Jr, Moon JE. Pharmacotherapies for lipid modification: beyond the statins. Nat Rev Cardiol. 2013;10:560-570.

45. Catapano AL. Perspectives on low-density lipoprotein cholesterol goal achievement. Curr Med Res Opin. 2009;25:431-447.

46. Jones P, Nair R, Thakker KM. Prevalence of dyslipidemia and lipid goal attainment in statin-treated subjects from 3 data sources: a retrospective analysis. J Am Heart Assoc. 2012;1:e001800.

47. Reiner Z. Resistance and intolerance to statins. Nutr Metab Cardiovasc Dis. 2014;24:1057-1066.

48. Reiner Z, Catapano AL, De Backer G, et al. ESC Committee for Practice Guidelines (CPG) 2008-2010 and 2010-2012 Committees. ESC/EAS Guidelines for the management of dyslipidaemias: the Task Force for the management of dyslipidaemias of the European Society of Cardiology (ESC) and the European Atherosclerosis Society (EAS). Eur Heart J. 2011;32:1769-1818.

49. Stone NJ, Robinson JG, Lichtenstein AH, et al. Treatment of Blood Cholesterol to Reduce Atherosclerotic Cardiovascular Disease Risk in Adults: Synopsis of the 2013 ACC/AHA Cholesterol Guideline. Ann Intern Med. 2014;160:339-343.
50. Raal FJ, Giugliano RP, Sabatine MS, et al. PCSK9 monoclonal antibody evolocumab (AMG 145): a pooled analysis of over 1300 reduction in lipoprotein(a) with the patients in 4 phase 2 trials. J Am Coll Cardiol. 2014;63:1278-1288.

51. Nordestgaard BG, Chapman MJ, Ray K, et al. Lipoprotein(a) as a cardiovascular risk factor: current status. Eur Heart J. 2010;23: 2844-2853.

52. Tavori H, Melone M, Rashid S. Alirocumab: PCSK9 inhibitor for LDL cholesterol reduction. Exp Rev Cardiovasc Ther. 2014;12: 1137-1144.

53. Cicero AF, Tartagni E, Ertek S. Safety and tolerability of injectable lipid-lowering drugs: a review of available clinical data. Exp Opin Drug Saf. 2014;13:1023-1030

54. Cannon CP, Cariou B, Blom D, et al. Efficacy and safety of alirocumab in high cardiovascular risk patients with inadequately controlled hypercholesterolaemia on maximally tolerated doses of statins: the ODYSSEY COMBO II randomized controlled trial. Eur Heart J. Epub 2015 Feb 16.

55. Kastelein JJ, Robinson JG, Farnier M, et al. Efficacy and safety of alirocumab in patients with heterozygous familial hypercholesterolemia not adequately controlled with current lipid-lowering therapy: design and rationale of the ODYSSEY FH studies. Cardiovasc Drugs Ther. 2014;28(3):281-289.

56. Schwartz GG, Bessac L, Berdan LG, et al. Effect of alirocumab, a monoclonal antibody to PCSK9, on long-term cardiovascular outcomes following acute coronary syndromes: rationale and design of the ODYSSEY outcomes trial. Am Heart J. 2014;168(5):682-689.

57. Amgen. Open Label Study of Long Term Evaluation Against LDL-C Trial-2 (OSLER-2). Available from: https:/clinicaltrials.gov/show/ NCT01854918. NLM identifier: NCT01854918. Accessed May 07, 2015 .

58. Amgen. GLobal Assessment of Plaque reGression With a PCSK9 antibOdy as Measured by intraVascular Ultrasound (GLAGOV). Available from: https://clinicaltrials.gov/ct2/show/NCT01813422. NLM identifier: NCT01813422. Accessed May 07, 2015.

59. Amgen. Further Cardiovascular Outcomes Research With PCSK9 Inhibition in Subjects With Elevated Risk (FOURIER). Available from: https://clinicaltrials.gov/ct2/show/NCT01764633. NLM identifier: NCT01764633. Accessed May 07, 2015.

60. Regeneron Pharmaceuticals. Study to Evaluate the Efficacy and Safety of an Every Four Weeks Treatment Regimen of Alirocumab (REGN727/ SAR236553) in Patients With Primary Hypercholesterolemia (ODYSSEY CHOICE 1). Available from: https://clinicaltrials.gov/ ct2/show/NCT01926782. NLM identifier: NCT01926782. Accessed May 12, 2015.
Drug Design, Development and Therapy

\section{Publish your work in this journal}

Drug Design, Development and Therapy is an international, peerreviewed open-access journal that spans the spectrum of drug design and development through to clinical applications. Clinical outcomes, patient safety, and programs for the development and effective, safe, and sustained use of medicines are a feature of the journal, which

\section{Dovepress}

has also been accepted for indexing on PubMed Central. The manuscript management system is completely online and includes a very quick and fair peer-review system, which is all easy to use. Visit http://www.dovepress.com/testimonials.php to read real quotes from published authors. 